\title{
Cuidado à mulher em situação de violência doméstica: representações de enfermeiras(os) hospitalares*
}

\author{
Daniele Ferreira Acosta ${ }^{1}$, Vera Lúcia de Oliveira Gomes ${ }^{2}$, Denize Cristina Oliveira ${ }^{3}$, Sergio Corrêa Marques ${ }^{4}$, \\ Giovana Calcagno Gomes ${ }^{5}$
}

\footnotetext{
* Extraído da Tese de Doutorado em Enfermagem "Representações sociais de enfermeiras hospitalares acerca da violência doméstica contra a mulher e sua relação com o cuidado" defendida no Programa de PósGraduação em Enfermagem da Universidade Federal do Rio Grande, em 2015.

${ }^{1}$ Enfermeira, Doutora em Enfermagem. Professora Adjunta da Universidade Federal do Rio Grande. Rio Grande, RS, Brasil. E-mail: nieleacosta@gmail.com.

${ }^{2}$ Enfermeira, Doutora em Enfermagem. Professora Titular da Universidade Federal do Rio Grande. Rio Grande, RS, Brasil. E-mail: vlog1952@gmail.com.

${ }^{3}$ Enfermeira, Doutora em Saúde Pública. Professor Titular da Universidade do Estado do Rio de Janeiro. Rio de Janeiro, RJ, Brasil. Email: dcuerj@gmail.com.

${ }^{4}$ Enfermeiro, Doutor em Enfermagem. Professor Adjunto da Universidade do Estado do Rio de Janeiro. Rio de Janeiro, RJ, Brasil. Email: sergiocmarques@uol.com.br.

${ }^{5}$ Enfermeira, Doutora em Enfermagem. Professor Adjunto da Universidade Federal do Rio Grande. Rio Grande, RS, Brasil. E-mail: giovanacalcagno@furg.br.
}

Recebido: 22/07/2016.

Aceito: 07/02/2017.

Publicado: 18/09/2017.

\section{Como citar esse artigo:}

Acosta DF, Gomes VLO, Oliveira DC, Marques de violência doméstica: representações de enfermeiras(os) hospitalares. Rev. Eletr. Enf. [Internet]. 2017 [acesso em: ____;19:a21. Disponível em: http://dx.doi.org/10.5216/ree.v19.42471. $\mathrm{SC}$, Gomes GC. Cuidado à mulher em situação

\section{RESUMO}

Estudo objetivou analisar a estrutura e os conteúdos das representações sociais de enfermeiras(os) acerca do cuidado à vítima de violência doméstica. Participaram enfermeiras(os) de dois hospitais públicos de médio porte do município do Rio Grande/RS. Os dados foram colhidos por meio de evocações livres frente ao termo indutor "cuidado à vítima" e analisados por software. As(os) enfermeiras(os) possuem as representações centradas no cuidado psicossocial demonstrado pelos termos presentes no núcleo central. $\mathrm{Na}$ zona de contraste infere-se a presença de um subgrupo que reconhece a importância do cuidado físico, sem se limitar a ações tecnicistas. Na periferia evidencia-se a face com o trabalho frente aos termos assistência e orientação. Profissionalismo revela a necessidade de abordar o objeto com base no saber reificado. Considera-se que por se tratar de um tema com carga emocional, somado à hospitalização e a fragilidade da vítima, o enfoque psicossocial é mais significativo no contexto do cuidado.

Descritores: Violência Doméstica; Cuidados de Enfermagem; Saúde da Mulher; Assistência Hospitalar.

\section{INTRODUÇÃO}

A violência doméstica contra a mulher (VDCM) é um fenômeno antigo, que tem ganhado maior visibilidade pela frequência e gravidade dos casos. Tal reconhecimento deve-se, em parte, aos noticiários que divulgam aqueles com desfecho de morte ou com lesões físicas graves. Situações como essas costumam despertar interesse e discussões entre a população, bem como entre os profissionais da saúde que assistem 
a essa clientela demonstrando, atualmente, uma postura mais proativa e positiva nesse contexto ${ }^{(1)}$.

Por outro lado, as situações com menor potencial ofensivo à mulher são banalizadas ou silenciadas no $\operatorname{lar}^{(2)}$ ou, ainda, invisibilizadas nos serviços de saúde sem o devido diagnóstico pelos profissionais ${ }^{(1,3)}$, inibindo a sequência de um atendimento interdisciplinar e intersetorial à vítima. Independentemente da forma de violência, as sequelas imediatas ou tardias exigem o seu reconhecimento como necessidade de intervenção na área da saúde.

Pesquisas nacionais e internacionais buscam dimensionar o fenômeno através da caracterização do perfil das vítimas e dos agressores ${ }^{(4-5)}$, da identificação das formas de violência ${ }^{(4,6)}$, das consequências ocasionadas à saúde da mulher ${ }^{(6-8)}$, bem como das estratégias de enfrentamento ${ }^{(9)}$, mas raras são as que discorrem sobre o cuidado.

Sabe-se que as consequências da violência não se restringem à dimensão física, mental, sexual e reprodutiva; incluem-se o ônus com medicamentos, com a alta rotatividade no emprego, com a perda de produtividade. Frente a magnitude da violência doméstica a Organização Mundial da Saúde a classificou como um problema global de saúde pública com proporções epidêmicas ${ }^{(10)}$, antes encarado apenas como uma problemática social.

Diante disso, destaca-se a importância dos profissionais da saúde, não somente na identificação dos casos, mas também frente às ações de prevenção e promoção à saúde. Todavia, são reconhecidas as fragilidades de caráter técnico-profissional ${ }^{(11-13)}$ e organizacional ${ }^{(11-13)}$, presentes tanto na área da saúde, quanto na jurídica e policial. Além disso, por tratar-se de um fenômeno histórico-cultural, o atendimento à mulheres vitimadas pode ser permeado por crenças, rotulações e pré-julgamentos que acabam inibindo uma atuação profissional efetiva.

Foi evidenciado em estudo, com profissionais da saúde, o julgamento moral no manejo dos casos de violência sexual, por meio de argumentos como: "Dizem que foi estupro, mas tinham usado droga", "Muitas querem fazer aborto e arrumam esta desculpa"(11). A culpabilização da mulher reflete na sua revitimização, configurando uma prática que vai de encontro à ferramenta de trabalho do enfermeiro, o cuidado.

O cuidado é um misto da dimensão técnica aliada aos princípios humanísticos, que se expressa em gestos, emoções, envolvimento, ação e responsabilidade. É colocado em prática quando se estabelece uma relação significativa com o ser que o recebe, no sentido de promover, manter, ou recuperar não somente a integridade física, mas a social, a emocional, a espiritual e a intelectual ${ }^{(14)}$. Portanto, é considerado descuido profissional as ações puramente técnicas, sem interação e reflexão sobre a realidade em que se insere a vítima, sujeito proativo na construção deste cuidado.

No contexto da violência doméstica é fundamental o acolhimento, a orientação sobre a rede de apoio auxiliando a mulher no processo decisório de maneira consciente e autônoma. No entanto, raras são as pesquisas que discorrem sobre o significado do cuidado à mulher vitimada dando voz ao profissional sobre essa prática no cenário hospitalar. Assim, a elaboração deste estudo se justifica na medida em que a análise das representações sociais de enfermeiras, acerca do cuidado às mulheres vitimadas, poderá facilitar a 
compreensão da influência desses conteúdos nas práticas de cuidado e saúde. Com isso, utilizou-se a Teoria das Representações Sociais, pois permite captar, interpretar e analisar a assimilação conceitual elaborada pelas enfermeiras acerca do objeto.

Essas evocações, verbalizadas, carregam as crenças, as atitudes, as práticas, os sentimentos dos sujeitos, tanto individual, quanto coletivo, permitindo um olhar consensual sobre a realidade. Portanto, este estudo busca analisar a estrutura e os conteúdos das representações sociais de enfermeiras acerca do cuidado à mulher vítima de violência doméstica.

\section{MÉTODOS}

Estudo qualitativo, fundamentado na Teoria das Representações Sociais, em sua Abordagem Estrutural ou Teoria do Núcleo Central ${ }^{(15)}$. Essa teoria consiste na identificação de um Núcleo Central (NC), que está relacionado à memória coletiva e confere significado à representação e de um Sistema Periférico, mais associado às características individuais e ao contexto imediato ${ }^{(15)}$.

Foram informantes do estudo enfermeiras(os) que trabalhavam em dois hospitais públicos de médio porte do município do Rio Grande/RS. Para tanto, selecionaram-se as unidades de clínica médica, clínica cirúrgica e traumatológica, unidade materno-obstétrica, unidade de terapia intensiva, centro de queimados e pronto-socorro, pela maior probabilidade de internação de mulheres vítimas de violência doméstica. De cada uma dessas unidades convidou-se até três enfermeiras(os), com tempo mínimo de dois meses de atuação no referido setor, por se considerar que com esse tempo tivessem tido a oportunidade de prestar cuidados a vítimas de violência.

A coleta de dados, realizada no período compreendido entre maio/agosto de 2014, foi efetuada por meio da Técnica de Evocações Livres que consiste em solicitar aos sujeitos que enunciem palavras ou expressões que vêm imediatamente à cabeça diante de um termo indutor, nesse caso "cuidado à vítima". Esse método permite a apreensão das cognições de maneira descontraída e espontânea, que podem ser mascaradas nas produções discursivas ${ }^{(16)}$. O registro das evocações, enunciadas pelos participantes, foi escrito manualmente pela própria pesquisadora em sala disponível e reservada nas unidades.

No tratamento das evocações buscou-se identificar e evitar a polissemia do material coletado, uniformizando termos similares, masculino/feminino, plural/singular, e reduzindo frases ou expressões longas. Na digitação do corpus foram incluídas todas as regras de preparo, bem como a ordem de evocação das palavras. Para a análise das evocações utilizou-se um software, o qual realiza análise estatística das palavras segundo a frequência e a ordem média de evocação (O.M.E), servindo de base para a construção do Quadro de Quatro Casas.

Nesse quadro, o NC situa-se no quadrante superior esquerdo e é formado pelos termos evocados com maior frequência e mais prontamente. Sua determinação é essencialmente social, ligada à memória coletiva e à história do grupo, é insensível ao contexto imediato e estabilizador das representações. A zona de contraste, no quadrante inferior esquerdo, é formada por palavras pouco evocadas, porém nas primeiras 
posições e comporta elementos que podem expressar variações das representações advindas de subgrupos. A periferia, situada à direita, é mais flexível que o NC, pois sofre influência da realidade, comporta contradições e manifestações da heterogeneidade do grupo ${ }^{(14)}$.

O projeto foi aprovado pelo Comitê de Ética em Pesquisa na Área da Saúde da Universidade Federal do Rio Grande sob o parecer no 80/2014.

\section{RESULTADOS}

Participaram do estudo 96 enfermeiras e quatro enfermeiros, com idade entre 24 e 59 anos. Mais de $60 \%$ tinham até três anos de trabalho na atual unidade. As unidades de atuação foram as relacionadas à assistência clínica, cirúrgica, materno-obstétrica, intensiva, traumatológica, além do centro de queimados e pronto socorro.

No que se refere às evocações diante do termo indutor "cuidado à vítima" obteve-se um total de 486 palavras, sendo 61 diferentes. Isso significa que o corpus analisado é homogêneo, considerando a baixa frequência de palavras distintas. Das evocações, 100 palavras foram enunciadas na primeira posição, 100 na segunda, 98 na terceira e na quarta e 88 na quinta posição.

Assim, a frequência média das palavras foi de 22 e a frequência mínima foi de 13. A O.M.E foi de três, indicando que a maioria das palavras foi evocada entre a segunda e a terceira posição, em um ranking de até cinco. Quanto mais próximas do um, mais significado a palavra confere às representações. A distribuição dos termos evocados, no Quadro de Quatro Casas, possibilitou a análise da estrutura e dos conteúdos das representações, formada pelo núcleo central, zona de contraste e elementos periféricos (1aㅡ e 2a periferia).

Tabela 1: Quadro de quatro casas formado pela evocação das enfermeiras ao termo indutor “cuidado à vítima”. Rio Grande, RS, Brasil, 2014.

\begin{tabular}{|c|c|c|c|c|c|c|}
\hline Freq. Méd. & O.M.E & & & \multirow{3}{*}{ orientação } & & \multirow{3}{*}{3,133} \\
\hline \multirow{4}{*}{$\geq 22$} & cuidado psicossocial & 41 & 2,122 & & \multirow{2}{*}{20} & \\
\hline & apoio & 33 & 2,818 & & & \\
\hline & proteção & 29 & 2,828 & assistência & 26 & 3,192 \\
\hline & atenção & 24 & 2,625 & & & \\
\hline \multirow{4}{*}{$<22$} & acolhimento & 20 & 2,250 & cuidado & 16 & 3,688 \\
\hline & cuidado físico & 16 & 2,938 & encorajar & 16 & 3,250 \\
\hline & \multirow{2}{*}{ ouvir } & \multirow{2}{*}{14} & \multirow{2}{*}{2,143} & respeito & 14 & 3,143 \\
\hline & & & & profissionalismo & 13 & 3,846 \\
\hline
\end{tabular}

\section{Elementos do Núcleo Central}

Destaca-se como elemento do NC o termo cuidado psicossocial que englobou enunciações relativas ao cuidado psicológico, moral e social. Trata-se de um elemento com possível centralidade nas representações, por ser evocado mais prontamente e com maior importância quantitativa.

Como prováveis elementos nucleares também se destacam apoio, proteção e atenção. Cada termo abarca conteúdos que se direcionam as ações tanto à vítima quanto à família. Deste modo, o NC aponta representações do cuidado à vítima, essencialmente pragmática, ou seja, quanto à necessidade de práticas 
profissionais voltadas à dimensão emocional e intelectual.

\section{Elementos da Zona de Contraste}

No quadrante inferior esquerdo, que correspondente à Zona de Contraste, foram identificados os termos acolhimento, cuidado físico e ouvir. Chama atenção o termo cuidado físico, diante de seu contraste em relação ao NC. Infere-se que os sujeitos envolvidos nestas enunciações possuem uma cognição centrada nas lesões físicas e na higiene corporal, conforme evidenciado na padronização dos termos. Os termos acolhimento e ouvir são cognições muito associadas ao aspecto relacional do cuidado, mostrando que a dimensão física não limita o diálogo, a escuta, o envolvimento com o outro.

\section{Elementos Periféricos}

Na Primeira Periferia, situada no quadrante superior direito, figuram os elementos orientação e assistência. Cabe destacar que ambos apresentaram saliência na expressão do universo representacional, com 30 e 26 aparições, respectivamente, porém foram evocados mais tardiamente, ultrapassando a O.M.E com valor igual a três, adotado como corte para elegibilidade prototípica à centralidade na estrutura das representações.

Incluem-se naquela denominação conteúdos alusivos ao esclarecimento da vítima quanto aos seus direitos; à informação sobre a denúncia policial, sobre recidivas, sobre a violência, bem como aos próprios cuidados. Já o termo assistência remete a necessidade de atendimento multidisciplinar, de um ambiente preparado para a manutenção da vida, além de procedimentos e rotinas frente o processo de cuidar.

Quanto à Segunda Periferia, localizada no quadrante inferior esquerdo, foram identificados os elementos cuidado; profissionalismo; respeito, evocado mais prontamente, seguido de encorajar. Essa designação comporta conteúdos acerca do encorajamento à denúncia do agressor, à mudança e reflexão sobre a vida e à autoestima. Profissionalismo, segundo as produções verbais, revela aspectos relacionados às características e às competências da enfermagem diante do cuidado à mulher vitimizada.

\section{DISCUSSÃO}

$\mathrm{Na}$ análise geral dos conteúdos que compõem as representações sociais do cuidado às vítimas de violência doméstica evidenciam-se cinco enfoques do cuidado. No quadrante superior esquerdo situa-se o enfoque psicossocial representado pelo termo cuidado psicossocial. Os outros elementos nucleares reforçam esse enfoque do cuidado corroborando que não é, apenas, a presença maciça de um elemento que define a sua centralidade, mas também a cognição qualitativa que confere significado à representação ${ }^{(15)}$.

A determinação do NC é essencialmente social, ligada à memória coletiva e à história do grupo ${ }^{(16)}$. Assim, observa-se que o cuidado relacional se estabelece como prioridade no atendimento à vítima de violência doméstica, remetendo à características da profissão. A essência da Enfermagem é o cuidar, que implica um movimento direcionado a algo ou a alguém que é alvo de interesse, sendo manifestado quando 
envolve sensibilidade, afeto e compaixão ${ }^{(17)}$.

Além de tudo, trata-se de uma "ação moral para satisfazer, aliviar, ajudar, confortar, apoiar", favorecer o bem do outro ${ }^{(14)}$. Nesse sentido, os termos apoio e atenção revelam um conteúdo avaliativo socioafetivo no cuidado às vítimas. Considerando que o cuidado não é exatamente uma técnica, como um curativo, mas a preocupação, o interesse, a motivação; esses termos configuram a interação entre profissional-cliente, reforçando o lado humanístico do cuidado, bem como a esfera subjetiva dos profissionais. Nessa concepção, somam-se o apoio institucional representado pela necessidade de amparo judicial, social e familiar à vítima.

O suporte prestado por meio dos serviços voltados às mulheres em situação de violência tem sido destacado como coadjuvante para que a mulher consiga sair do ciclo violento, somado à problematização de gênero, das normas que justificam o controle masculino e das políticas referentes aos direitos da mulher ${ }^{(6,9,18)}$. A palavra atenção, enunciada com menor frequência, remete a valorização da mulher e da queixa, bem como a dedicação no processo de cuidar. É importante que as(os) enfermeiras(os) reconheçam a rede de apoio local para que possam instrumentalizar as mulheres sobre os seus direitos, contribuindo para uma tomada de decisão consciente.

Com base no enfoque psicossocial como centro das representações é possível inferir que as(os) enfermeiras(os) entendem que esse cuidado é mais significativo perante os transtornos ocasionados à mulher. Transtornos esses que não podem ser sanados apenas com procedimento técnico; pois trata-se da "dor da alma", da cicatriz que marca uma vida de sonhos, não sendo facilmente tratada, nem mesmo curada, o que requer maior atenção. Por outro lado, existe uma concepção dos trabalhadores da saúde de que esse problema é pertinente aos psicólogos pela sua formação clínica, reduzindo, assim, o cuidado apenas ao encaminhamento a esses profissionais ${ }^{(19)}$.

No quadrante inferior esquerdo situam-se os termos ouvir, evocado mais prontamente e acolhimento, o mais frequente, evidenciando que acolher, dar prioridade no atendimento, promover espaço de escuta são fundamentais no cuidado à vítima. O acolhimento, enquanto postura, reforça as relações; pois envolve o escutar e tratar de forma humanizada. Ainda que os serviços de saúde não sejam especializados no atendimento às mulheres em situação de VDCM, muitas vezes, constituem o primeiro local de procura, sendo $\mathrm{a}(\mathrm{o})$ enfermeira(o) a(o) profissional que assume papel primordial no acolhimento, pois é responsável pela triagem no âmbito hospitalar, espaço onde recebe vítimas de violência, além de permanecer 24 horas em contato com as clientes.

No entanto, estudos divulgam algumas fragilidades que permeiam as práticas profissionais, comprometendo o acolhimento das vítimas. Essas se referem à insegurança, ao medo, ao despreparo para abordar as situações ${ }^{(3,13,20)}$ ou ainda à culpabilização da mulher e a justificativas relacionadas ao excesso de trabalho ${ }^{(11)}$. Para tanto, é necessário encarar os problemas sociais como sendo do âmbito da saúde, assumindo práticas que permitam a produção de novos modos de cuidado ${ }^{(21)}$.

O cuidado físico integra o segundo enfoque e, por situar-se na zona de contraste, pode revelar a existência de um subgrupo minoritário portador de representação diferente ${ }^{(16)}$. Esse elemento 
contranormativo remete ao cuidado do corpo, diferentemente dos significados presentes no NC. Discursos de profissionais revelam a identificação da violência quando se apresenta de maneira explícita, ou seja, pelas marcas físicas, além do predomínio da abordagem centrada nas lesões ou disfunções orgânicas ${ }^{(19,22)}$. É comum valorizarem as queixas físicas, no processo de cuidar e fundamentarem-se nos processos biológicos.

A incorporação desse enfoque nas representações pode ser influenciada pelo contexto profissional das(os) enfermeiras(os). A maioria dos casos que chegam às unidades de urgência e emergência ou que necessitam de hospitalização são aqueles relacionados aos ferimentos com arma de fogo e arma branca ${ }^{(23)}$. Embora se trate de lesões físicas graves, o envolvimento da equipe de saúde treinada permite uma assistência resolutiva que contemple as necessidades biopsicossociais da mulher vitimada, sem se limitar aos agravos físicos. O cuidado só é concretizado quando o cuidador se envolve e se compromete com o ser cuidado de forma interativa ${ }^{(17)}$.

Na primeira periferia, quadrante superior direito, os termos orientação e assistência expressam outros enfoques do cuidado; o educativo e o técnico, respectivamente. Esses elementos periféricos das representações expressam, claramente, as práticas profissionais revelando que o cuidado à vítima busca um bem físico, psicológico e social. No conjunto das evocações referentes à orientação, inclui-se o esclarecimento à vítima sobre os seus direitos, o aconselhamento, a orientação sobre o autocuidado, sobre as recidivas e a denúncia do agressor. Observa-se, assim, de maneira mais intrínseca, o caráter preventivo do fenômeno e a influência da realidade frente o termo indutor, característica própria do sistema periférico.

A enfermagem utiliza os conhecimentos para alicerçar uma prática educativa, visando a reflexão, necessária para que os sujeitos possam cuidarem-se e fazerem escolhas conscientes. Ressalta-se que a indissociabilidade entre o cuidar e o educar é paradigma para o desenvolvimento profissional ${ }^{(14)}$. Além disso, a educação em saúde representa um importante instrumento que possibilita uma visão crítica e participativa, da vítima, contribuindo para a promoção de sua saúde ${ }^{(24)}$.

O termo assistência remete à técnica, com certa preocupação na organização institucional e no modelo de atendimento às mulheres, que inclui os exames, as sorologias, os procedimentos e equipe interdisciplinar, conforme evidenciado nas evocações que foram padronizadas. Esses conteúdos fazem parte do processo de cuidar, não podendo ser encarado apenas como cuidado curativista, pois são necessários.

Salienta-se que a assistência diferencia-se do cuidado no que tange as ações desempenhadas, naquele caso, voltado às tarefas. A primeira não necessariamente inclui o cuidar/cuidado, pois pode-se não estar cuidando no sentido pleno que envolva responsabilidade, interesse e desvelo ${ }^{(14)}$. Sabe-se que a técnica é muito valorizada na enfermagem, todavia, o cuidado é com e para o outro; é mais do que a intervenção no outro.

O atendimento às vítimas, por equipe multiprofissional, é indispensável, mas precisa ser integrado para que tenha eficácia. É essencial que haja comunicação entre os profissionais e articulação intersetorial dos serviços a fim de possibilitar o enfrentamento da violência contra a mulher. A conjugação de diferentes saberes e fazeres permite aos profissionais a tomada de decisões sem se limitar a divisão das necessidades 
biopsicossociais, possibilitando transformá-las em um cuidado holístico ${ }^{(25)}$.

Na segunda periferia, no quadrante inferior esquerdo, situam-se os elementos cuidado, encorajar, profissionalismo e respeito evocados mais tardiamente e com menor frequência. O encorajar é inerente ao processo de cuidar, forma como se dá o cuidado, e vai ao encontro do enfrentamento da violência doméstica. A palavra respeito, evocada mais prontamente nesse quadrante, se concretiza na maneira de interagir com o paciente; ouvindo-o, sendo honesto e sincero.

O termo profissionalismo revela o quinto enfoque expressando as características e as competências do profissional diante do cuidado à mulher em situação de violência. Desponta como atributos a necessidade de agir com ética, de disponibilizar-se, de ter conhecimento, responsabilidade e visão sem críticas, marcando as dimensões éticas e estéticas do cuidado. Tal termo refere-se ao julgamento valorativo do profissional e por integrar os conteúdos e a estrutura das representações sociais do cuidado, revela a necessidade de abordar o objeto estudado com base no saber reificado e não somente por meio da subjetividade de cada profissional.

Estudos têm divulgado a utilização de práticas rotineiras ${ }^{(19)}$ e o despreparo da equipe de saúde no atendimento à vítimas de violência doméstica ${ }^{(2,20)}$, na qual acabam estabelecendo uma atuação pessoal, distanciada da atenção qualificada ${ }^{(3)}$. Tal achado reforça as evidências deste estudo, ou seja, da necessidade de educação permanente frente ao objeto em tela.

\section{CONCLUSÃO}

As representações das(os) enfermeiras(os), acerca do cuidado às mulheres vítimas de violência doméstica, encontram-se fortemente ancoradas em práticas que primam pelo bem-estar psicossocial. Tal fato remete às características da Enfermagem, fundamentada nos princípios de amor, sensibilidade, doação e preocupação com o ser cuidado.

Esse resultado sinaliza para uma concepção ampliada acerca do cuidado à vítima de violência doméstica, que também inclui o indispensável cuidado físico, presente nas representações. No cenário de atuação das(os) enfermeiras(os) a interface com a hospitalização das mulheres vitimadas parece não refletir numa conduta técnica, isolada, e sim associada à necessidade de apoiar a mulher a tomar decisões, de encorajar a saída do ciclo de violência, bem como orientação sobre a rede de apoio primária e secundária.

Diante do impacto físico e emocional às vítimas, ocasionados pelas agressões, é importante que sejam acolhidas no serviço de saúde, assim como evidenciado, evitando sua revitimização. 0 enfoque educativo reflete no caráter preventivo do fenômeno conferindo significado às representações e a preocupação dos profissionais com a saúde da mulher e com o seu projeto de vida.

Cabe destacar que as representações centralizadas no aspecto psicossocial também podem ser decorrentes do fato da maioria ser do sexo feminino, que por vezes se colocam no lugar das vítimas. Desta forma, como limitação cita-se tanto o aspecto regionalizado e temporal do estudo, quanto a impossibilidade de comparar as representações entre enfermeiras e enfermeiros perante a disparidade entre o número de 
informantes de ambos os sexos.

Este estudo também aponta para a relação entre práticas de cuidado à mulheres em situação de violência doméstica e a capacitação, face a realidade vivenciada, com base nas produções verbais relacionadas ao profissionalismo.

De maneira geral, esses achados contribuem com a comunidade científica na medida em que não encerram a abordagem sobre o assunto, lançando desafios para aprofundar se são as representações que influenciam as práticas ou se são essas que interferem na representação.

\section{Agradecimentos}

Agradecimentos à Fundação de Amparo à Pesquisa do Estado do Rio Grande do Sul pela concessão de bolsa no doutorado.

\section{REFERÊNCIAS}

1. Vieira EM, Ford NJ, De Ferrante FG, Almeida AM, Daltoso D, Santos MA. The response to gender violence among Brazilian health care professionals. Cien Saude Colet [Internet]. 2013 [acesso em: 11 set. 2017];18(3):681-90. Disponível em: http://dx.doi.org/10.1590/S1413-81232013000300014.

2. Fonseca DH, Ribeiro CG, Leal NSB. Violência doméstica contra a mulher: realidades e representações sociais. Psicol Soc [Internet]. 2012 [acesso em: 11 set. 2017];24(2):307-14. Disponível em: http://dx.doi.org/10.1590/S010271822012000200008.

3. Gomes NP, Erdmann AL, Bettinelli LA, Higashi GDC, Carneiro JB, Diniz NMF. Significado da capacitação profissional para o cuidado da mulher vítima de violência conjugal. Esc Anna Nery [Internet]. 2013 [acesso em: 11 set. 2017];17(4):683-9. Disponível em: http://dx.doi.org/10.5935/1414-8145.20130012.

4. Acosta DF, Gomes VLO, Barlem ELD. Perfil das ocorrências policiais de violência contra a mulher. Acta Paul Enferm [Internet]. 2013 [acesso em: 11 set. 2017];26(6):547-53. Disponível em: http://dx.doi.org/10.1590/S010321002013000600007.

5. Sanz-Barbero B, Rey L, Otero-García L. Estado de salud y violencia contra la mujer en la pareja. Gac Sanit [Internet]. 2014 [acesso em: 11 set. 2017];28(2):102-8. Disponível em: http://dx.doi.org/10.1016/j.gaceta.2013.08.004.

6. Calvo González G, Camacho Bejarano R. La violencia de género: evolución, impacto y claves para su abordaje. Enfermería Glob [Internet]. 2014 [acesso em: 11 set. 2017];(33):424-39. Disponível em: http://dx.doi.org/10.6018/eglobal.13.1.181941.

7. Arruda CN, Braide ASG, Nations M. "Carne crua e torrada": a experiência do sofrimento de ser queimada em mulheres nordestinas, Brasil. Cad Saude Publica [Internet]. 2014 [acesso em: 11 set. 2017];30(10):2057-67. Disponível em: http://dx.doi.org/10.1590/0102-311X00175713.

8. Chepuka L, Taegtmeyer M, Chorwe-Sungani G, Mambulasa J, Chirwa E, Tolhurst R. Perceptions of the mental health impact of intimate partner violence and health service responses in Malawi. Glob Health Action [Internet]. 2014 [acesso em: 11 set. 2017];7(1):24816. Disponível em: https://dx.doi.org/10.3402/gha.v7.24816.

9. Menezes PRM, Lima IS, Correia CM, Souza SS, Erdmann AL, Gomes NP. Enfrentamento da violência contra a mulher: articulação intersetorial e atenção integral. Saude soc [Internet]. 2014 [acesso em: 11 set. 2017];23(3):778-86.

Disponível em: http://dx.doi.org/10.1590/S0104-12902014000300004.

10. World Health Organization. Violence against women: a 'global health problem of epidemic proportions' [Internet]. 2013 [acesso em: 11 set. 2017]. Disponível em:

http://www.who.int/mediacentre/news/releases/2013/violence_against_women_20130620/en/

11. Villela WV, Vianna LAC, Lima LFP, Sala DCP, Vieira TF, Vieira ML, et al. Ambiguidades e contradições no atendimento de mulheres que sofrem violência. Saude soc [Internet]. 2011 [acesso em: 11 set. 2017];20(1):113-23. Disponível em: http://dx.doi.org/10.1590/S0104-12902011000100014. 
12. Barros LA, Albuquerque MCS, Gomes NP, Riscado JLS, Araújo BRO, Magalhães JRF. The (un)receptive experiences of female rape victims who seek healthcare services. Rev Esc Enferm USP [Internet]. 2015 [acesso em: 11 set. 2017];49(2):193-200. Disponível em: http://dx.doi.org/10.1590/\$0080-623420150000200002.

13. Lira CEPR, Silva PPAC, Trindade RFC. Conduta dos agentes comunitários de saúde diante de casos de violência familiar. Rev. Eletr. Enf. [Internet]. 2012 [acesso em: 11 set. 2017];14(4):928-36. Disponível em: https://doi.org/10.5216/ree.v14i4.12237.

14. Waldow V R. Bases e princípios do conhecimento e da arte da enfermagem. Petrópolis: Vozes; 2008. 15. Abric JC. A abordagem estrutural das representações sociais. In: Moreira ASP, Oliveira DC. Estudos interdisciplinares de representação social. Goiânia: AB; 2000. p. 27-38.

16. Oliveira DC, Marques SC, Gomes AMT, Teixeira MCTV. Análise das evocações livres: uma técnica de analise estrutural das representações sociais. In: Moreira ASP. Perspectivas teórico-metodológicas em representações sociais. João Pessoa: UFPE Ed. Universitária; 2005. p. 573-603.

17. Waldow VR, Borges RF. Caring and humanization: relationships and meanings. Acta Paul Enferm [Internet]. 2011 [acesso em: 11 set. 2017];24(3):414-8. Disponível em: http://dx.doi.org/10.1590/S0103-21002011000300017. 18. Heise LL, Kotsadam A. Cross-national and multilevel correlates of partner violence: an analysis of data from population-based surveys. Lancet Glob Heal [Internet]. 2015 [acesso em: 11 set. 2017];3(6):e332-40. Disponível em: http://dx.doi.org/10.1016/S2214-109X(15)00013-3.

19. Silva EB, Padoin SMM, Vianna LAC. Violence against women and care practice in the perception of the health professionals. Texto contexto - enferm [Internet]. 2015 [acesso em: 11 set. 2017];24(1):229-37. Disponível em: http://dx.doi.org/10.1590/0104-07072015003350013.

20. Reis MJ, Lopes MHBM, Higa R, Turato ER, Chvatal VLS, Bedone AJ. Vivências de enfermeiros na assistência à mulher vítima de violência sexual. Rev Saude Publica [Internet]. 2010 [acesso em: 11 set. 2017];44(2):325-31. Disponível em: http://dx.doi.org/10.1590/S0034-89102010000200013.

21. Silva SS, Assis MMA. Family health nursing care: weaknesses and strengths in the Unified Health System. Rev Esc Enferm USP [Internet]. 2015 [acesso em: 11 set. 2017];49(4):603-9. Disponível em: http://dx.doi.org/10.1590/S0080623420150000400010.

22. Bernz IM, Coelho EBS, Lindner SR. Desafio da violência doméstica para profissionais da saúde: revisão da literatura. Saúde \& Transformação Social [Internet]. 2012 [acesso em: 11 set. 2017];3(3):105-11. Disponível em: http://incubadora.periodicos.ufsc.br/index.php/saudeetransformacao/article/view/1545.

23. Ilha MM, Leal SMC, Soares JSF. Mulheres internadas por agressão em um hospital de pronto socorro: (in)visibilidade da violência. Rev Gaucha Enferm [Internet]. 2010 [acesso em: 11 set. 2017];31(2):328-34. Disponível em: http://dx.doi.org/10.1590/S1983-14472010000200018.

24. Cervera DPP, Parreira BDM, Goulart BF. Educação em saúde: percepção dos enfermeiros da atenção básica em Uberaba (MG). Cien Saude Colet [Internet]. 2011 [acesso em: 11 set. 2017];16(suppl 1):1547-54. Disponível em: http://dx.doi.org/10.1590/S1413-81232011000700090.

25. Santos MA, Vieira EM. Recursos sociais para apoio às mulheres em situação de violência em Ribeirão Preto, SP, na perspectiva de informantes-chave. Interface (Botucatu) [Internet]. 2011 [acesso em: 11 set. 2017];15(36):93-108. Disponível em: http://dx.doi.org/10.1590/S1414-32832011000100008. 